\title{
Comparative serum proteomic analysis involving liver organ-specific metastasis-associated proteins of nasopharyngeal carcinoma
}

\author{
CHANGCHUAN PAN ${ }^{1,3}$, YALAN TAO $^{2}$, MING ZHAO $^{1}$, WANG LI ${ }^{1}$, ZILIN HUANG $^{1}$, \\ JING GAO $^{2}$, YANHEN WU ${ }^{4}$, JINGRUI YU ${ }^{3}$, PEIHONG WU ${ }^{1}$, YUNFEI XIA ${ }^{2}$ and JIN LU $^{3}$ \\ Departments of ${ }^{1}$ Medical Imaging and Interventional Radiology, and ${ }^{2}$ Radiation Oncology, \\ State Key Laboratory of Oncology in South China, Sun Yat-Sen University Cancer Center; ${ }^{3}$ Medical Oncology, \\ Sichuan Cancer Hospital and Institute, The Second People's Hospital of Sichuan Province; \\ ${ }^{4}$ The First Affiliated Hospital of Jinan University, Guangzhou, Sichuan, P.R. China
}

Received December 19, 2011; Accepted February 6, 2012

DOI: $10.3892 /$ etm.2012.526

\begin{abstract}
Metastasis is the main cause of cancer-related mortality; patients with liver metastases (LM) have the worst prognosis among patients with nasopharyngeal carcinoma (NPC). However, at present, few biomarkers for detecting organ-specific metastasis have been identified. Proteomics, an ultra-sensitive analytical technique, can detect molecular changes before organ-specific metastasis occurs. Analysis with matrix-assisted, laser desorption-ionization time-of-flight mass spectrometry (MALDI-TOF-MS), combined with magnetic chemical affinity beads is a new technique for evaluating protein separation. We sought to identify potential liver-specific, metastasis-associated proteomic printing in patients with NPC. We examined 64 serum samples from 50 patients who had pathologically confirmed NPC and 14 who had pathologically confirmed non-NPC with LM using MALDI-TOF-MS with weak cation bead protein chips. During follow-up of at least 37 months (maximum, 176 months) following radiotherapy, we confirmed 16 cases of LM (LM NPC), 16 cases without LM (non-LM NPC) and 18 cases without metastasis (non-M NPC). Using comparison analysis, 4 protein mass peaks, 4155.34, 4194.87, 4210.78 and $4249.56 \mathrm{~m} / \mathrm{z}$ were identified as liver-specific, metastasis-associated protein peaks in NPC and
\end{abstract}

Correspondence to: Professor Yunfei Xia, Department of Radiation Oncology, State Key Laboratory of Oncology in South China, Sun Yat-Sen University Cancer Center, 651 Dongfeng Road East, Guangzhou 510060, P.R. China

E-mail: xiayunfei@yahoo.com

Dr Peihong Wu, Department of Medical Imaging and Interventional Radiology, State Key Laboratory of Oncology in South China, Sun Yat-Sen University Cancer Center, 651 Dongfeng Road East, Guangzhou 510060, P.R. China

E-mail:wuph@sysucc.org.cn

Key words: nasopharyngeal carcinoma, organ-specific metastasis, liver metastasis, proteomics two of them $(4155$ and $4249 \mathrm{~m} / \mathrm{z})$ met two different statistical criteria in both ClinProt software analyses and discriminant analyses. Models based on the 4 potential serum markers of NPC discriminated between LM NPC, non-LM NPC, non-M NPC and non-NPC LM analyzed with sieved markers. The recognition capability and cross-validation of these models for differentiating the above 4 groups are all approximately $80 \%$. MALDI-TOF-MS combined with tree analysis models may provide a clinical diagnostic platform for detecting potential liver-specific, metastasis-associated proteomic printing in NPC. However, markedly differential proteins still need to be identified.

\section{Introduction}

In numerous tumor types, distant metastases are the major cause of cancer-related deaths. Metastasis is a complex process involving several steps: local invasion, adhesion, migration, survival in the blood or lymphatic system and extravasation, colonization of distant organs and growth into tumors. However, cancers exhibit distinct patterns of organ-specific metastases. Multiple organs may be seeded, but metastatic tumors may grow in only one or a few of these organs (1). This non-random process is dependent on multiple interactions of specific metastatic cells with the organ-specific microenvironment (2). Scientific understanding of metastatic spread is limited, and the molecular mechanisms causing particular characteristics of metastases are largely unknown. In patients with metastatic nasopharyngeal carcinoma (NPC), the prognosis is generally poor. Despite modern intensity-modulated radiotherapy, approximately $20 \%$ of patients with stage M0 disease still experience distant metastases within 3 years after completing treatment, and the total incidence of distant metastases is between 16 and 42\% (3). Median survival of patients with metastatic NPC is only 11-18 months after metastases have been identified (4-7). NPC usually metastasizes to the lung, bone and liver. Final prognosis is dependent on the involved organ. Patients with liver metastasis (LM) have poorer survival (3-5 months) than patients with metastases 
Table I. Clinical characteristics and long-term follow-up data for 50 patients with pathologically confirmed nasopharyngeal carcinoma (NPC), with and without distant metastases.

\begin{tabular}{|c|c|c|c|}
\hline Characteristics & Non-M NPC (n=18) & LM NPC $(n=16)$ & Non-LM NPC $(\mathrm{n}=16)$ \\
\hline Age, median (range) in years & $44.7(32-61)$ & $45.1(18-71)$ & $45.4(21-65)$ \\
\hline Gender, male/female & $13 / 5$ & $10 / 6$ & $12 / 4$ \\
\hline \multicolumn{4}{|l|}{ T stage (1998) } \\
\hline $\mathrm{T} 1$ & 1 & 0 & 1 \\
\hline $\mathrm{T} 2$ & 5 & 4 & 3 \\
\hline $\mathrm{T} 3$ & 7 & 7 & 11 \\
\hline $\mathrm{T} 4$ & 5 & 5 & 1 \\
\hline \multicolumn{4}{|l|}{ N stage (1998) } \\
\hline N0 & 3 & 2 & 0 \\
\hline N1 & 5 & 4 & 5 \\
\hline $\mathrm{N} 2$ & 7 & 6 & 8 \\
\hline N3 & 3 & 4 & 3 \\
\hline \multicolumn{4}{|l|}{ Histological classification (WHO) } \\
\hline I & 0 & 1 & 0 \\
\hline II & 8 & 7 & 10 \\
\hline III & 10 & 8 & 6 \\
\hline \multicolumn{4}{|l|}{ Presentation of metastases } \\
\hline Synchronous/metachronous & - & $0 / 16$ & $1 / 15$ \\
\hline Mean follow-up time (months) & 87 & 82 & 93 \\
\hline Survival status (alive/deceased) & $18 / 0$ & $1 / 15$ & $3 / 13$ \\
\hline Median overall survival (months) & - & 33.0 & 36.72 \\
\hline Median metastatic survival (months) & - & 14.3 & 21.17 \\
\hline
\end{tabular}

non-M NPC, NPC patients without distant metastasis; LM NPC, NPC patients with liver metastasis; non-LM NPC, NPC patients with non-liver distant metastasis.

in other organs (6-13 months) $(4,7,8)$. Knowledge concerning the molecular mechanisms of liver-specific metastasis in NPC could help guide a clincian towards efficient treatment and assist in the prediction of final outcome. Among several proteomic studies on NPC (9-11), few involved markers of metastasis (12) and none specifically address the characteristics of liver metastasis. We investigated whether the behavior of liver metastases is related to changes in plasma protein markers in patients with NPC.

\section{Materials and methods}

This retrospective comparative proteomic study was approved by the Institutional Blood Sample Library of the Sun Yat-sen University Cancer Center.

Patients. Valid records of the Sun Yat-Sen University Cancer Center were searched for patients with a histological diagnosis of NPC whose serum samples had been obtained at least 3 years prior. The requirement for blood sample collection more than three years prior was required, as most NPC patients of distant metastases, including liver metastases, are identified within 3 years after completion of radiotherapy $(4,7)$.
Distant metastases were identified via histological evaluation or imaging combined with a subsequent clinical follow-up examination. Patient clinical data were collected and survival status was verified on 31 August 2010 by direct telecommunication with the patient or patient's family and verification of clinic attendance records. The present study included 50 NPC patients (Table I, Fig. 1): 18 cases without distant metastasis (non-M NPC group), 16 patients with liver metastasis (LM NPC group) and 16 patients with non-liver distant metastasis (non-LM NPC group) prior to the cut-off follow-up day. To create a control group, serum samples were collected, as described below, from 14 patients with LM from other pathologically confirmed forms of cancer (LM non-NPC group). Of these 14 patients, 11 had colorectal cancer, 1 had pancreatic cancer, 1 had breast cancer and 1 had ovarian cancer.

Blood sample preparation. In the present study, all serum samples from NPC patients with metastasis were obtained between January 2000 and July 2007. Samples from 18 cases of non-M NPC were obtained prior to verifiable distant metastasis during the same period.

Blood samples were collected and processed according to a standardized protocol: samples were collected in $8.5-\mathrm{ml} \mathrm{BD}$ 
Vacutainer SST tiger-top tubes and $1 \mathrm{~h}$ later were centrifuged at $1400-2000 \mathrm{x} \mathrm{g}$ at room temperature for $10 \mathrm{~min}$. Serum (supernatant) was transferred to 4-ml cryovials, with $1 \mathrm{ml}$ in each and stored at $-80^{\circ} \mathrm{C}$ until analysis.

Plasma protein fractionation. All plasma samples were thawed and purified using a reagent set with chemically coated magnetic beads (weak cation-coated, Bruker Daltonics Co., Billerica, MA). As previously described (13), serum (2 $\mu \mathrm{l})$ was incubated with $5 \mu \mathrm{l}$ of magnetic beads for $10 \mathrm{~min}$ on a ClinProt robotic platform (Bruker Daltonics Co.) according to the manufacturer's specifications. Unbound proteins were discarded and each sample was washed twice in binding buffer. Briefly, samples were purified through binding, washing and elution, according to the manufacturer's suggested protocol. A total of $5 \mu$ of each sample was eluted and the purified plasma was further diluted 8-fold with the elution solution in preparation for mass spectrometry analysis.

Mass spectrometry profiling of the plasma proteome. For matrix-assisted, laser desorption-ionization, time-of-flight mass spectrometry (MALDI-TOF-MS) analysis, $1 \mu \mathrm{l}$ of the above-mentioned diluted plasma was mixed with $0.5 \mu \mathrm{l}$ of matrix solution containing $2 \mathrm{~g} / \mathrm{l} \alpha$-cyano-4-hydroxycinnamic acid and $1 \%$ formic acid in 50\% acetonitrile and the droplet was allowed to dry on the MALDI sample plate (AnchorChip, Bruker Daltonics Co.). Mass spectra were obtained with an Ultraflex MALDI-TOF-MS (Bruker Daltonics Co.) operated in either positive ion linear or reflectron mode, depending on the analysis being performed. Profiling data were acquired in linear mode geometry and mass maps were acquired in reflectron mode $(13,14)$. All spectra were obtained randomly over the surface of the matrix spot. The profiling spectra were calibrated externally using a mixture of protein and peptide standards (Bruker Daltonics Co.). A \pm 2 Da mass accuracy for each spectrum was observed and was likely due to differences in sample geometry on the plate surface. Briefly, all spectra were processed by automatic baseline subtraction, peak detection, recalibration and peak area calculation according to predefined settings. Each spectrum was the result of 400 laser pulses per $\mathrm{m} / \mathrm{z}$ segment per sample delivered in four sets of 100 pulses (at $50 \mathrm{~Hz}$ ) to each of four different locations on the surface of the spot.

The criteria for protein mass peak detection $(\mathrm{m} / \mathrm{z})$ were as follows: signal-to-noise ratio (S/N) $>5$, a 2-Da peak width filter, and a maximum peak number of 200. The intensities of the peaks of interest were normalized with the peak intensity of an ACTH internal standard. More than 10\% of the molecular weight was sieved in simultaneous samples, with the discrepancy of identical spinnacle in different samples $<0.3 \%$ after removal of the initial data noise.

Identifying potential liver-specific, metastasis-associated protein masses. According to the seed and soil theory of metastasis and a recent report on the molecular basis of metastasis $(2,15)$, potential proteomic printing was sorted into seven groups of plasma markers related to: Factor 0, the absence of distant metastasis from any cancer; Factor 1 , the initiation and progression of metastasis; Factor 2, liver-specific metastases; Factor 3, non-liver distant metastases; Factor 4, liver-specific

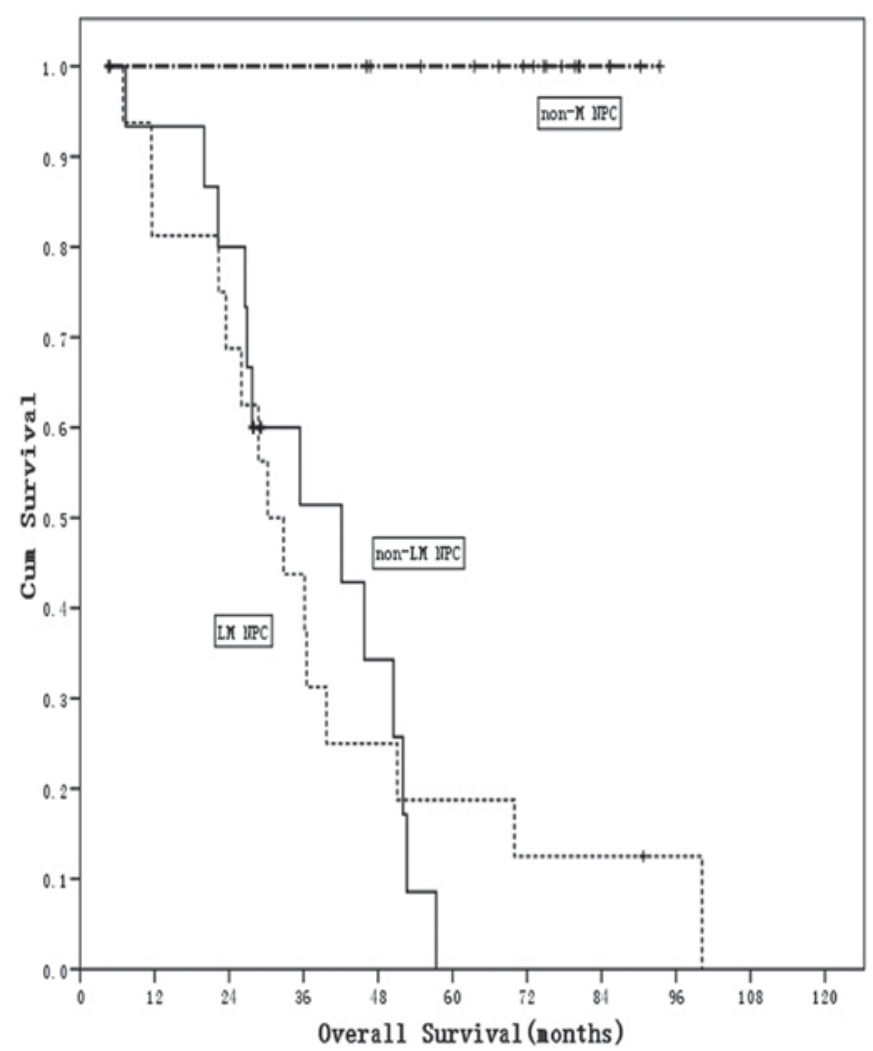

Figure 1. Overall survival of 50 patients with pathologically confirmed nasopharyngeal carcinoma, with and without distant metastases. LM NPC, NPC patients with liver metastasis; non-LM NPC, NPC patients with non-liver distant metastasis; non-M NPC, NPC patients without distant metastasis.

metastases from NPC; Factor 5, non-liver distant metastases from NPC; Factor 6, liver-specific metastases from non-NPC.

To verify the claim that the peaks identified are clearly associated with liver-specific metastasis, an attempt to identify peaks with this comparative analysis of serum proteomic of the groups was conducted (Fig. 2). Subsequently, the differential expression of the protein mass peaks was analyzed by an alternative statistical approach, discriminant analysis.

Statistical methods. Each spectrum obtained from MALDITOF-MS was analyzed by ClinProt software version 2.0 (Bruker Daltonics Co.). When differential expression of the protein mass peaks was identified between any two groups, these data were imported into the ClinProt soft ware. Expression of the protein mass peaks was considered to be significant at a P-value of $<0.05$. Each serum sample was processed at least twice to confirm the results and to reduce bias. In the last step, a commonly used shared nearest neighbors approach was applied in order to obtain alternative estimates of the diagnostic potential of combining all peaks. After each diagnostic model was generated, a $20 \%$ leave out cross-validation process was performed.

\section{Results}

As shown in Fig. 2, 28 protein mass peaks from 2000 to $20000 \mathrm{~m} / \mathrm{z}$ between the LM NPC and non-M NPC groups were identified, as well as 9 significant protein mass peaks 
A Study population

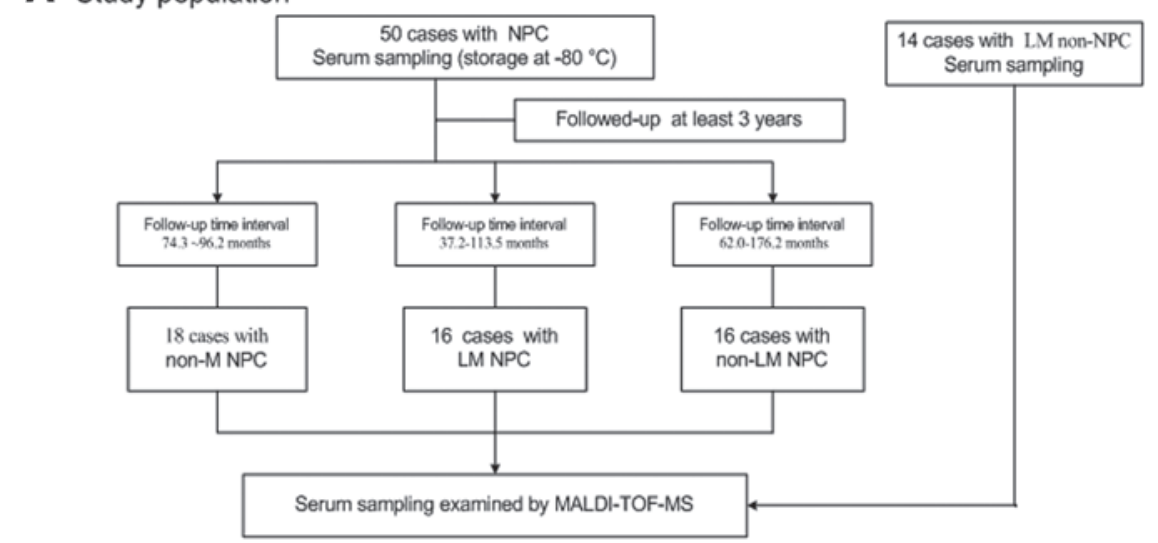

B Comparative analysis

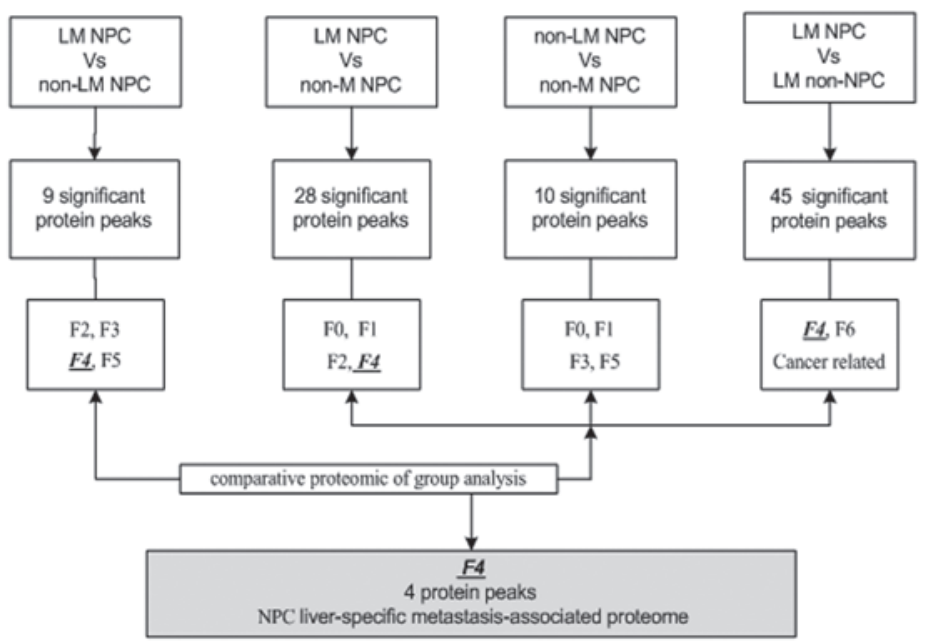

Figure 2. Visual summary of the (A) study population and (B) the comparisons made in a study using proteomic analysis to identify NPC liver organ-specific metastasis-associated plasma markers. Factor 0 (F0), plasma markers related to patients without distant metastasis; Factor 1 (F1), plasma markers related to patients with metastasis; Factor 2 (F2), plasma markers related to patients with liver-specific metastasis; Factor 3 (F3), plasma markers related to patients with distant metastasis to sites other than the liver; Factor 4 (F4), plasma markers related to patients with liver-specific metastasis from nasopharyngeal carcinoma; Factor 5 (F5), plasma markers related to patients with non-liver distant metastasis from nasopharyngeal carcinoma; Factor 6 (F6), plasma markers related to patients with liver-specific metastasis from non-nasopharyngeal carcinoma.

Table II. Mass-to-charge ratios for protein mass peaks that differentiate between patients who have nasopharyngeal carcinoma with and without distant metastases.

\begin{tabular}{lccc}
\hline Protein mass peaks & $\begin{array}{c}\text { Patients without } \\
\text { distant metastases }(\mathrm{n}=18)\end{array}$ & $\begin{array}{c}\text { Patients with } \\
\text { liver metastases }(\mathrm{n}=16)\end{array}$ & $\begin{array}{c}\text { Patients without } \\
\text { liver metastases }(\mathrm{n}=16)\end{array}$ \\
\hline 4155, mean $(\mathrm{SD}) \mathrm{m} / \mathrm{z}$ & $33.72(13.52)$ & $19.28(11.50)$ & $7.71(6.56)$ \\
P-value & - & 0.00214 & $2.4 \mathrm{E}-5$ \\
4194, mean (SD) $\mathrm{m} / \mathrm{z}$ & $83.63(37.05)$ & $47.61(21.94)$ & $25.53(21.43)$ \\
P-value & - & 0.00199 & $6.84 \mathrm{E}-6$ \\
4210, mean (SD) $\mathrm{m} / \mathrm{z}$ & $425.30(212.65)$ & $236.37(120.77)$ & $126.44(116.6)$ \\
P-value & - & 0.00158 & $9.18 \mathrm{E}-5$ \\
4249, mean $(\mathrm{SD}) \mathrm{m} / \mathrm{z}$ & $33.72(17.14)$ & $19.28(17.14)$ & $7.71(8.94)$ \\
P-value & - & 0.00158 & $2.4 \mathrm{E}-5$
\end{tabular}

of protein between LM NPC and non-LM NPC groups, and so on. By comparing the LM NPC and LM non-NPC groups, one can identify not only cancer-specific serum peptides (NPC and non-NPC related markers) but also proteins associated with cancer-specific and organ-specific metastases (Factor 4, Factor 6). Also by comparing the LM NPC and non-LM NPC 


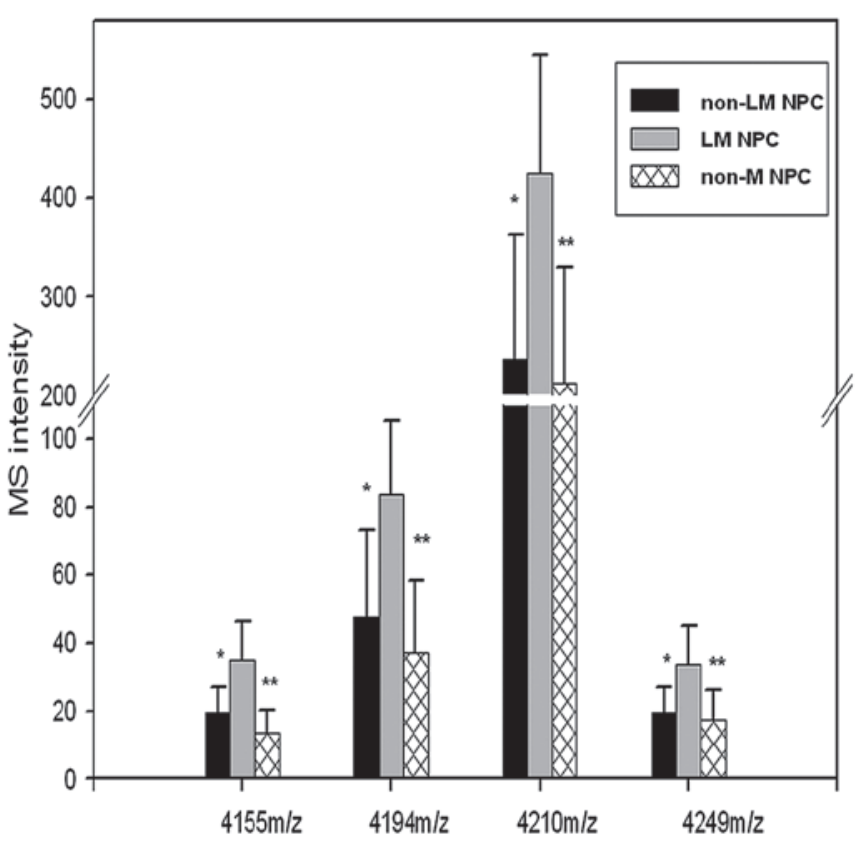

Distribution of NPC-specific markers

Figure 3 . Four protein mass peaks $(\mathrm{m} / \mathrm{z})$ that differed signifcantly among the patients with nasopharyngeal carcinoma, with liver metastasis (LM NPC) and non-liver distant metastasis (non-LM NPC), as well as without distant metastasis (non-M NPC). ${ }^{*} \mathrm{P}<0.05,{ }^{* * *} \mathrm{P}<0.001$.

groups, cancer-specific serum peptides, that is NPC-related markers, and common markers related to metastasis initiation and progression (Factor 1) could be deleted, but NPC- and organ-specific, metastasis-associated peptides (Factors 2-5) could be identified. It is valid that Factor 4 cannot be obtained by comparing the non-LM NPC and the non-M NPC groups (Fig. 2). After discriminant and comparison analysis, Factors 4 including 4 protein mass peaks $(4155.34,4194.87,4210.78$ and $4249.56 \mathrm{~m} / \mathrm{z}$ ) were identified as NPC liver-specific, metastasisassociated protein peaks (Fig. 1). The value in the LM NPC group was significantly higher than that of the non-LM NPC and the non-M NPC groups (Table II, Fig. 3). These four protein mass peaks were not detected in comparisons between the non-LM NPC and non-M NPC groups. The decision tree differentiated between the LM NPC and non-LM NPC groups and was built up, and 3 final crunodes consisting of 4155, 4249 and $4123 \mathrm{~m} / \mathrm{z}$ were identified (Fig. 4). Two ( 4155 and $4249 \mathrm{~m} / \mathrm{z}$ ) of these above four protein mass peaks fulfilled two different statistical criteria in both ClinProt software analyses and discriminant analyses.

The above four protein mass peaks were randomly chosen to ClinProt software by optimization in order to establish the combined diagnostic model for differentiating between the LM NPC group from the non-LM NPC group (Table III). The recognition capability of the diagnostic model for differentiation of the LM NPC group from the non-LM NPC group was found to be $100 \%$ for both groups. The diagnostic accuracy of cross-verification was $80.5 \%$ for the LM NPC group and $79 \%$ for the non-LM NPC group. After deletion of the 4210.78 or the $4194.87 \mathrm{~m} / \mathrm{z}$ protein mass peaks in the above 4 peaks, the

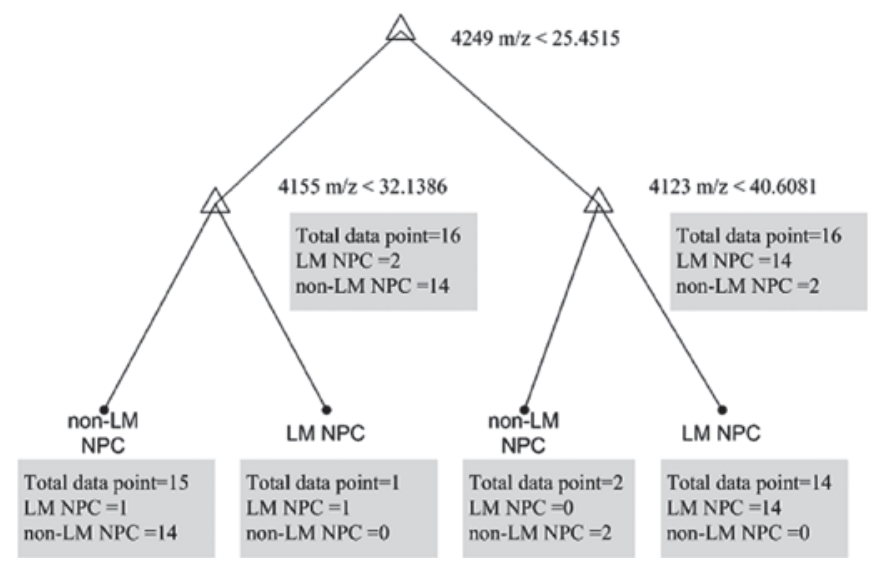

Figure 4. Models of serum protein mass peaks that discriminate between patients who have nasopharyngeal carcinoma with and without liver metastases. NPC, nasopharyngeal carcinoma; LM NPC, NPC patients with liver metastasis; non-LM NPC, NPC patients with non-liver distant metastasis; LM non-NPC, patients with liver metastasis but not from NPC.

remaining 3 protein mass peaks were again chosen to establish the combined diagnostic model for differentiation between the LM NPC from the non-LM NPC groups. The accuracy for differentiating the LM NPC group from the non-LM NPC group of the diagnostic models was once again found to be $100 \%$ for both groups. The accuracy of cross-verification was between 74.2 and approximately $80.5 \%$ for the LM NPC and between 73.3 and approximately $77.8 \%$ for the non-LM NPC groups.

The combined diagnostic model for differentiation between the LM NPC and non-M NPC, as well as the diagnostic model for differentiation between the LM NPC from LM non-NPC groups, were set up as shown in Table III. The recognition capability and accuracy of cross-verification was between 80 and approximately $100 \%$.

\section{Discussion}

Progression to distant metastasis is a critical point in the disease course of patients with solid epithelial malignancies, such as NPC. The standard clinical process for discovery of distant metastasis is mostly based on imaging. The early seeding of metastatic cells into the bloodstream is usually not identified by radiography or scans such as CT and MRI. Also, an ideal serum tumor marker has yet to be identified for monitoring the early metastasis of NPC. Such molecular markers need to be identified, not only for detecting early metastases, but also for classifying disease and developing appropriate treatments.

Advancements in genomics, proteomics and bioinformatics have improved our understanding of the cause, carcinogenesis and progression of the disease (16). Proteomic profiling is based upon the fact that proteins represent the dynamic state of the cells, reflecting earlier pathophysiological changes in the disease more accurately than genomic sequencing (17). Proteomic patterns should assist in the detection of tumor biomarkers, as well as in evaluating the efficacy of anticancer drugs. Unfortunately, the proteome associated with NPC distant metastasis is currently poorly understood. In this study, an extensive proteomic analysis of organ-specific metastasis 
in the serum of patients with NPC was performed. A standardized serum preparation method for MALDI-TOF-MS was utilized based on weak cation magnetic beads and was able to identify many valuable, low-abundance protein masses of interest. MALDI-TOF-MS is capable of detecting proteins that can aid in the diagnosis of many common types of cancer. Serum proteomics profiling may also help predict the response to treatment, in addition to improving our understanding of metastasis (18). Such analyses have identified several clues concerning the markers of metastasis. Zheng et al identified two serum protein biomarkers (9184.4 and $9340.9 \mathrm{~m} / \mathrm{z}$ ) useful for monitoring micro-metastases in colorectal cancer (19). Another recent study also associated a collection of membrane and membrane-associated proteins with colorectal cancer (20). Additionally, protein markers associated with lymph node metastases in colorectal and prostate cancer have been profiled $(21,22)$.

In a previous NPC study, assays of MALDI-TOF were used to detect 13 differentially expressed protein spots. Three potential NPC metastasis-specific serum biomarkers were further validated including sICAM-1, HSP70 and SAA, by comparing lymph node metastasis and non-lymph node metastasis groups (12). Two separate statistical approaches were used to search for serum biomarkers that were significantly associated with liver-specific metastasis and two proteins were identified (4155 and $4249 \mathrm{~m} / \mathrm{z}$ ) that met statistical criteria in both analyses. With this in mind, several other questions remain such as which protein and what functions of these identified protein masses were differentially expressed in our study. Also, what is the basis for organ-specific metastases associated with these proteins? As we know, three major theories have been proposed to explain these organ-specific metastases. According to the first theory, tumor cells exit the blood and lymphatic systems equally throughout all organs, but multiply only in those organs which have the appropriate growth factors. The second theory proposes that the endothelial cells that line blood vessels in target organs express adhesion molecules that cause circulating tumor cells to become attracted in those organs. Finally, the third theory of 'chemoattraction' holds that organ-specific attractant molecules enter the circulation, stimulating the migrating tumor cells to invade the walls of blood vessels and enter the organs. Therefore, the further identification of these liver-specific, metastasis-associated protein peaks may provide us with a better understanding of the events involved in liver colonization, NPC metastasis and provide a new mechanistic insight into organ-specific metastasis and the therapeutic potential for liver-specific metastasis in NPC. Jun et al identified (using MALDI-TOF-MS) 6 proteins which are differentially expressed in a lymph node metastatic prostate cancer group relative to a localized prostate cancer group which had been previously identified. These proteins, e-FABP5, MCCC2, PPA2, Ezrin, SLP2 and SM22, were further identified and validated as functionally relevant to cancer metastasis in tissue samples using real-time PCR, western blot analysis and immunohistochemistry staining.

Since the hematogeneous spread of tumor cells is considered to be a crucial event in metastasis, detecting serum biomarkers of this event could be of great clinical importance (17). In the present study, it was found that 4 serum protein mass peaks $(4155.34,4194.87,4210.78$ and $4249.56 \mathrm{~m} / \mathrm{z})$ 
differentiated LM NPC from non-LM NPC, which may be an NPC, liver-specific, metastasis-associated proteomic printing. Simultaneously, the three combined diagnostic models were also based on serum protein mass peaks that differed significantly between the LM NPC, non-LM NPC and LM non-NPC groups. The diagnostic models for differentiating LM NPC and non-M NPC had similar recognition accuracies when crossverified (70 to approximately $80 \%$ ) by deletion of the 4194.87 or $4210.78 \mathrm{~m} / \mathrm{z}$ protein mass peaks in the 4 significant protein mass peaks. As a result of these findings, there is evidence that the 4 serum protein mass peaks are useful diagnostic markers for the existence of LM NPC. In view of the metastatic heterogeneous nature of NPC and lack of valid methods for detecting early metastases in NPC, we believe our current proteomic approach has provided valuable information in the early differentiation between patients with NPC who have and who do not have liver metastases.

The difference in molecular weight between 4194.87 and $4210.78 \mathrm{~m} / \mathrm{z}$ is $16 \mathrm{~m} / \mathrm{z}$ - the molecular weight of oxygen - so one could postulate that these two protein mass peaks might be the same protein, with or without oxidation. This postulate needs verification through further experimentation.

In conclusion, through ClinProt software analysis and comparative proteomics of group analysis, we identified 4 serum mass spectrometry protein profiles among 50 patients with pathologically confirmed NPC who had at least 3 years of clinical follow-up data. These 4 protein peaks are potentially related to liver metastasis associated with NPC and are useful diagnostic markers for the existence of LM NPC.

\section{Acknowledgements}

We would like to thank Sun Yat-Sen University Cancer Center Blood Sample Library and Bioyong Technologies Inc. This study was supported by the grants from the Ministry of Science and Technology of China (No. 2006AA02Z4B4).

\section{References}

1. Husemann Y, Geigl JB, Schubert F, et al: Systemic spread is an early step in breast cancer. Cancer Cell 13: 58-68, 2008.

2. Chiang AC and Massague J: Molecular basis of metastasis. N Engl J Med 359: 2814-2823, 2008.

3. Kam MK, Teo PM, Chau RM, et al: Treatment of nasopharyngeal carcinoma with intensity-modulated radiotherapy: the Hong Kong experience. Int J Radiat Oncol Biol Phys 60: 1440-1450, 2004.

4. Hui EP, Leung SF, Au JSK, et al: Lung metastasis alone in nasopharyngeal carcinoma: a relatively favorable prognostic group - a study by the Hong Kong nasopharyngeal carcinoma study group. Cancer 101: 300-306, 2004.
5. Teo PM, Kwan WH, Lee WY, et al: Prognosticators determining survival subsequent to distant metastasis from nasopharyngeal carcinoma. Cancer 77: 2423-2431, 1996.

6. Wang CT, Cao KJ, Li Y, et al: Prognosis analysis of nasopharyngeal carcinoma patients with distant metastasis. Ai Zheng 26: 212-215, 2007.

7. Pan C, He N, Zhao M, et al: Subdividing the M1 stage of liver metastasis for nasopharyngeal carcinoma to better predict metastatic survival. Med Oncol 28: 1349-1355, 2011.

8. Huang CJ, Leung SW, Lian SL, et al: Patterns of distant metastases in nasopharyngeal carcinoma. Kaohsiung J Med Sci 12: 229-234, 1996.

9. Huang YJ, Xuan C, Zhang BB, et al: SELDI-TOF MS profiling of serum for detection of nasopharyngeal carcinoma. J Exp Clin Cancer Res 28: 85, 2009.

10. Chang KP, Wu CC, Chen HC, et al: Identification of candidate nasopharyngeal carcinoma serum biomarkers by cancer cell secretome and tissue transcriptome analysis: potential usage of cystatin A for predicting nodal stage and poor prognosis. Proteomics 10: 2644-2660, 2010

11. Wu CC, Chien KY, Tsang NM, et al: Cancer cell-secreted proteomes as a basis for searching potential tumor markers: nasopharyngeal carcinoma as a model. Proteomics 5: 3173-3182, 2005.

12. Liao Q, Zhao L, Chen X, et al: Serum proteome analysis for profiling protein markers associated with carcinogenesis and lymph node metastasis in nasopharyngeal carcinoma. Clin Exp Metastasis 25: 465-476, 2008.

13. Cheng AJ, Chen LC, Chien KY, et al: Oral cancer plasma tumor marker identified with bead-based affinity-fractionated proteomic technology. Clin Chem 51: 2236-2244, 2005.

14. Chang JT, Chen LC, Wei SY, et al: Increase diagnostic efficacy by combined use of fingerprint markers in mass spectrometry plasma peptidomes from nasopharyngeal cancer patients for example. Clin Biochem 39: 1144-1151, 2006.

15. Fokas E, Engenhart-Cabillic R, Daniilidis K, et al: Metastasis: the seed and soil theory gains identity. Cancer Metastasis Rev 26: 705-715, 2007.

16. Cho WC: Nasopharyngeal carcinoma: molecular biomarker discovery and progress. Mol Cancer 6: 1, 2007.

17. Hudler P, Gorsic M and Komel R: Proteomic strategies and challenges in tumor metastasis research. Clin Exp Metastasis 27: 441-451, 2010.

18. Liao CC, Mehta A, Ward NJ, et al: Analysis of post-operative changes in serum protein expression profiles from colorectal cancer patients by MALDI-TOF mass spectrometry: a pilot methodological study. World J Surg Oncol 8: 33, 2010.

19. Zheng GX, Wang CX, Qu X, et al: Establishment of serum protein pattern for screening colorectal cancer using SELDITOF-MS. Exp Oncol 28: 282-287, 2006.

20. Luque-Garcia JL, Martinez-Torrecuadrada JL, Epifano C, et al: Differential protein expression on the cell surface of colorectal cancer cells associated to tumor metastasis. Proteomics 10: 940-952, 2010

21. Pang J,Liu WP, Liu XP, et al: Profiling protein markers associated with lymph node metastasis in prostate cancer by DIGE-based proteomics analysis. J Proteome Res 9: 216-226, 2010.

22. Pei H, Zhu H, Zeng S, et al: Proteome analysis and tissue microarray for profiling protein markers associated with lymph node metastasis in colorectal cancer. J Proteome Res 6: 2495-2501, 2007. 